\title{
Presentation of Empirical Equations for Estimating Internal Friction Angle of GW and GC Soils in Mashhad, Iran Using Standard Penetration and Direct Shear Tests and Comparison with Previous Equations
}

\author{
Pouya Salari', Gholam Reza Lashkaripour ${ }^{2 *}$, Mohammad Ghafoori \\ ${ }^{1}$ Department of Geology, Ferdowsi University of Mashhad, International Branch, Mashhad, Iran \\ ${ }^{2}$ Department of Geology, Ferdowsi University of Mashhad, Mashhad, Iran \\ Email: "lashkaripour@um.ac.ir
}

Received 17 March 2015; accepted 2 May 2015; published 6 May 2015

Copyright $@ 2015$ by authors and Scientific Research Publishing Inc.

This work is licensed under the Creative Commons Attribution International License (CC BY).

http://creativecommons.org/licenses/by/4.0/

\section{(c) (i) Open Access}

\begin{abstract}
Presentation of empirical equations for estimating engineering properties of soils is a simple, low cost and widely-used method. One of the major concerns in using these equations is evaluating their accuracy in different conditions and regions which often lead to doubts about obtained results. Most of these equations were derived in special laboratories, different climate conditions and in soils with different geotechnical and geological engineering properties and were generalized to other conditions. The main question is that whether these methods are also applicable to other conditions. Using local equations and narrowing the usage range of various methods based on each region's properties are appropriate methods to solve these problems. This leads to simplified and faster analysis and high reliability in the obtained results. In this paper, empirical equations were derived to estimate internal friction angle, based on SPT numbers of Mashhad City's soils in Iran, using SPT and direct shear tests results from 50 samples (25 GW and 25 GC soil samples). The results showed similar values for predicted $\varphi$ values by SPT test and $\varphi$ values determined by direct shear tests.
\end{abstract}

\section{Keywords}

Internal Friction Angle, GW and GC Soil, Direct Shear Test, SPT Test

\footnotetext{
${ }^{*}$ Corresponding author.
}

How to cite this paper: Salari, P., Lashkaripour, G.R. and Ghafoori, M. (2015) Presentation of Empirical Equations for Estimating Internal Friction Angle of GW and GC Soils in Mashhad, Iran Using Standard Penetration and Direct Shear Tests and Comparison with Previous Equations. Open Journal of Geology, 5, 231-238. http://dx.doi.org/10.4236/ojg.2015.55021 


\section{Introduction}

Internal friction angle is one of the most important parameters in analyzing soil geotechnical properties and earthwork calculations. It has a wide range of applications such as calculating retaining walls, foundations, friction and end-bearing piles and so on [1] [2].

Based on properties of a given soil profile such as fine or coarse grained, various tests such as direct shear and triaxial tests are recommended for obtaining internal friction angle parameter. Although due to the soil disturbance during sampling as well as special laboratory conditions, these results may not completely represent true properties of soils and even in case of special care in doing the tests, they are still highly time-consuming and require using simpler empirical equations. This research aims to obtain internal friction angle of soils using standard penetration test for different types of soils in Mashhad. For this purpose, several equations have already been presented [3]. Internal friction angle for Mashhad city can be estimated using appropriate equations for the city soil conditions, soil types, samples depth and specific unit weight.

SPT number has been defined in various equations based on specific weight, grading, relative density, internal friction angle and undrained compressive strength [4] [5]. This number is also used for estimating bearing capacity of soil for foundation and elastic modulus calculations [6]. These equations and their approaches are often doubtful due to having a small amount of gathered data, lack of focus on special aspects or incorrect equations generalization [7]-[9].

Equations obtained by Shioi and Fukui (1982) are presented below (Equations (1) to (3)). Equation (1) is for roads and bridges, Equation (2) for buildings and Equation (3) is general.

$$
\begin{gathered}
\varnothing=\sqrt{N_{70}^{\prime}}+15 \\
\varnothing=0.36 N_{70}+27 \\
\varnothing=4.5 N_{70}+20 \text { (in general) }
\end{gathered}
$$

\section{FHWA Recommended Tables}

Federal Highway Administration recommend using Table 1 for correlating approximate SPT number, relative density and internal friction angle parameters with each other [10]. Following information is necessary to use Table 1:

1. Measured SPT numbers were obtained without any correction factors in field tests.

2. (Pa) is free sea level pressure.

3. Ranges in column (a) is based on Peck, Hanson, and Thornburn (1974) study.

4. Ranges in column (b) is based on Meyerhof (1956) study.

(3) Ranges in column (a) from Peck, Hanson, and Thornburn (1974).

(4) Ranges in column (b) and for CPT are from Meyerhof (1956).

Since the values are from field SPT tests, the table can be very useful and widely applicable [11].

\section{Methods of study}

First, SPT tests were carried out on 50 samples (25 GC and 25 GW samples in various depths). Results of direct shear tests ( $\varphi$ values) and also depth and dry unit weight of samples are shown in Table 2 and Table 3 [12]. These tables show in situ (SPT) and laboratory (direct shear) tests results. Locations of sampling in the city are shown in Figure 1.

Then, based on statistical validations, two equations were derived to estimate internal friction angle, based on SPT number for two soil types (GC, GW).

In order to use Table 2 and Table 3, some points must be considered.

1. Narrowing application range was done for special types of soils in order to achieve higher accuracy.

2. In order to attenuate the effects of some parameters such as weathering, all studied samples were taken from the depths of 4 to 15 meters.

3. In order to obtain better results, samples with special dry unit weight of 19 to $21 \mathrm{KN} / \mathrm{m}^{3}$ were considered.

4. Internal friction angle in these tables were obtained from direct shear tests.

5. All data were obtained from soil profiles on Vakilabad area located in the western part of Mashhad city. 
Table 1. Correlation between SPT and CPT results and friction angle of cohesionless soils (FHWA, 2003).

\begin{tabular}{|c|c|c|c|c|}
\hline & \multirow{2}{*}{ In-Situ Test Results } & \multirow{2}{*}{ Relative Density } & \multicolumn{2}{|c|}{$\varnothing($ degrees $)$} \\
\hline & & & $(a)^{3}$ & $(b)^{4}$ \\
\hline \multirow{5}{*}{$\begin{array}{l}\text { SPT N-Value } \\
\text { (blows/300 mm } \\
\text { or blows/ft) }\end{array}$} & 0 to 4 & Very Loose & $<28$ & $<30$ \\
\hline & 4 to 10 & Loose & 28 to 30 & 30 to 35 \\
\hline & 10 to 30 & Medium & 30 to 36 & 35 to 40 \\
\hline & 30 to 50 & Dense & 36 to 41 & 40 to 45 \\
\hline & $>50$ & Very Dense & $>41$ & $>45$ \\
\hline \multirow{5}{*}{$\begin{array}{l}\text { Normalized } \\
\text { CPT cone } \\
\text { bearing } \\
\text { resistance } \\
(\mathrm{qc} / \mathrm{Pa})\end{array}$} & $<20$ & Very Loose & \multicolumn{2}{|c|}{$<30$} \\
\hline & 20 to 40 & Loose & \multicolumn{2}{|c|}{30 to 35} \\
\hline & 40 to 120 & Medium & \multicolumn{2}{|c|}{35 to 40} \\
\hline & 120 to 200 & Dense & \multicolumn{2}{|c|}{40 to 45} \\
\hline & $>200$ & Very Dense & \multicolumn{2}{|c|}{$>45$} \\
\hline
\end{tabular}

Table 2. GW soils data obtained by laboratory and in-situ tests.

\begin{tabular}{|c|c|c|c|c|c|}
\hline Soil Type & Row & Dry Unit Weight $\left(\mathrm{KN} / \mathrm{m}^{3}\right)$ & Depth (m) & SPT Number & Internal Friction Angle \\
\hline & 1 & 19.5 & 13 & 38 & 34 \\
\hline & 2 & 20.1 & 11 & 39 & 34.4 \\
\hline & 3 & 19.6 & 10 & 41 & 34.9 \\
\hline & 4 & 19.9 & 14 & 45 & 37.2 \\
\hline & 5 & 20.8 & 6 & 42 & 36.2 \\
\hline & 6 & 20.1 & 9 & 40 & 35.5 \\
\hline & 7 & 20.0 & 13 & 38 & 34.2 \\
\hline & 8 & 19.2 & 4 & 39 & 34.3 \\
\hline & 9 & 19.6 & 14 & 39 & 34.5 \\
\hline & 10 & 20.6 & 9 & 43 & 36.8 \\
\hline & 11 & 20.3 & 5 & 42 & 36.3 \\
\hline & 12 & 19.1 & 6 & 45 & 37.3 \\
\hline \multirow[t]{13}{*}{ GW } & 13 & 19.8 & 8 & 42 & 36.4 \\
\hline & 14 & 20.4 & 11 & 43 & 36.7 \\
\hline & 15 & 20.9 & 10 & 38 & 34.1 \\
\hline & 16 & 20.1 & 14 & 45 & 37.2 \\
\hline & 17 & 19.3 & 13 & 43 & 36.9 \\
\hline & 18 & 20.6 & 12 & 41 & 36 \\
\hline & 19 & 19.2 & 9 & 42 & 36.1 \\
\hline & 20 & 19.7 & 13 & 42 & 36.3 \\
\hline & 21 & 19.6 & 11 & 43 & 36.4 \\
\hline & 22 & 20.7 & 10 & 44 & 37.1 \\
\hline & 23 & 19.3 & 8 & 40 & 35.8 \\
\hline & 24 & 20.6 & 14 & 38 & 34.2 \\
\hline & 25 & 20.4 & 11 & 44 & 37 \\
\hline
\end{tabular}


Table 3. GC soils data obtained by laboratory and in-situ tests.

\begin{tabular}{|c|c|c|c|c|c|}
\hline Soil Type & Row & Dry Unit Weight $\left(\mathrm{KN} / \mathrm{m}^{3}\right)$ & Depth (m) & SPT Number & Internal Friction Angle \\
\hline & 1 & 20.2 & 11 & 35 & 33 \\
\hline & 2 & 19.9 & 6 & 36 & 33.6 \\
\hline & 3 & 20.2 & 13 & 37 & 33.9 \\
\hline & 4 & 20.7 & 12 & 38 & 33.9 \\
\hline & 5 & 20.1 & 6 & 39 & 34.1 \\
\hline & 6 & 20.4 & 9 & 40 & 34.5 \\
\hline & 7 & 19.9 & 5 & 41 & 35 \\
\hline & 8 & 19.5 & 14 & 42 & 35.9 \\
\hline & 9 & 19.3 & 11 & 40 & 34.7 \\
\hline & 10 & 20.6 & 10 & 36 & 33.8 \\
\hline & 11 & 20.1 & 15 & 35 & 33.1 \\
\hline & 12 & 20.7 & 11 & 41 & 35.2 \\
\hline & 13 & 19.8 & 9 & 37 & 34.1 \\
\hline \multirow[t]{12}{*}{ GC } & 14 & 20.1 & 12 & 39 & 34.3 \\
\hline & 15 & 19.8 & 11 & 38 & 34.2 \\
\hline & 16 & 19.4 & 14 & 41 & 35.3 \\
\hline & 17 & 20.3 & 9 & 42 & 36.1 \\
\hline & 18 & 19.8 & 8 & 40 & 34.8 \\
\hline & 19 & 20.3 & 7 & 39 & 34.2 \\
\hline & 20 & 19.7 & 12 & 35 & 33.2 \\
\hline & 21 & 19.4 & 13 & 36 & 33.5 \\
\hline & 22 & 20.4 & 14 & 42 & 36.2 \\
\hline & 23 & 20.3 & 9 & 37 & 34.4 \\
\hline & 24 & 20.5 & 7 & 38 & 34.6 \\
\hline & 25 & 20.4 & 11 & 40 & 34.8 \\
\hline
\end{tabular}

\section{Results and Discussion}

Reliability and accuracy of the obtained equations must be measured by statistical reliability ratings. In order to obtain correlations between data, following steps were taken:

1. Drawing scatter diagram; Figure 2 and Figure 3 show scatter diagrams related to GC and GW soils properties.

2. Model fitting and obtaining coefficients: the aim of a proper model fitting is to determine correlation among control ( $x$ ) and response (y) variables (Equations (4)-(6) and Figure 2 and Figure 3) [13] [14].

$$
\begin{gathered}
y_{i}=\alpha+\beta x_{i}+e_{i} \\
y_{i}=\alpha+\beta \log x_{i}+e_{i} \\
y_{i}=\alpha+\exp \left(\beta x_{i}\right)+e_{i}
\end{gathered}
$$

3. Obtaining numerical value of Sig for comparing correlations. 


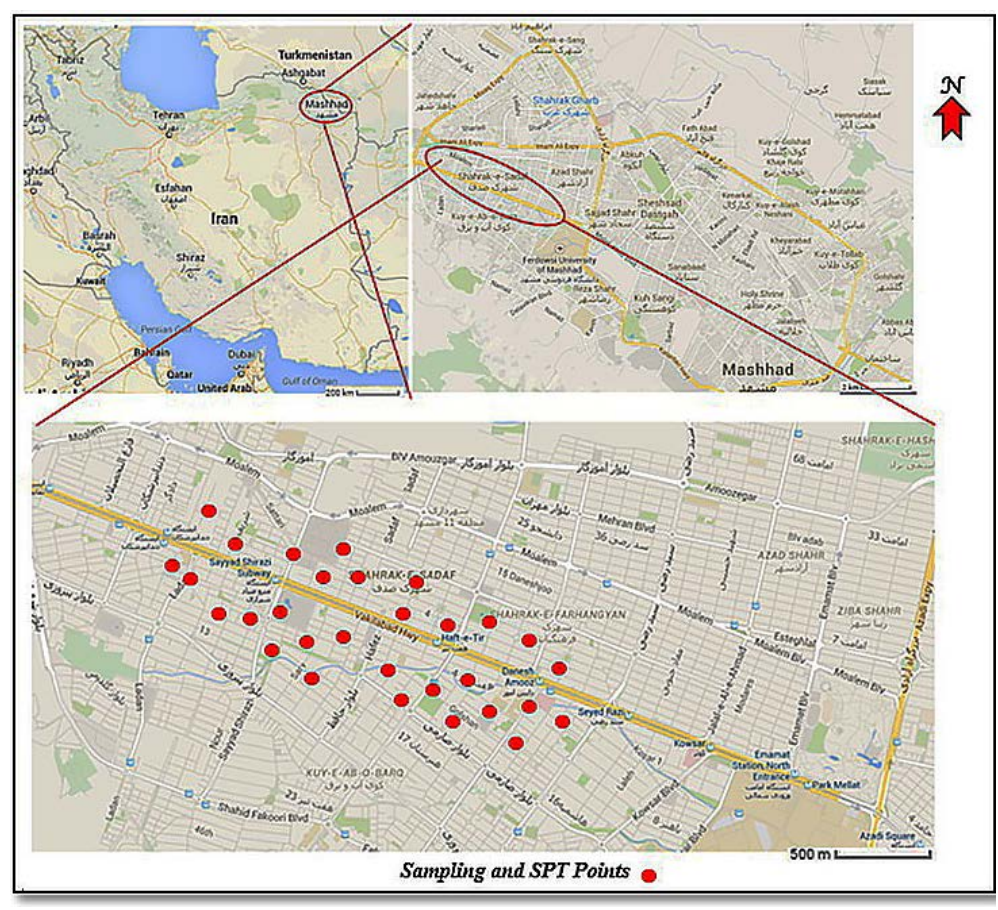

Figure 1. Locations of sampling and SPT testing in Mashhad city.

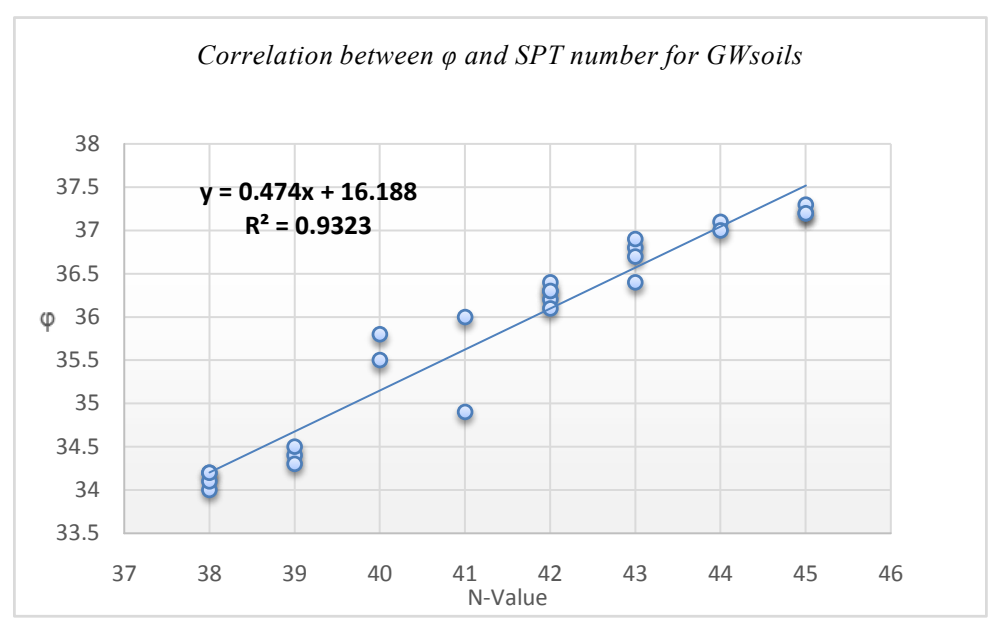

Figure 2. Correlation between $\varphi$ and SPT number for GW soils.

Models were studied on 95\% reliability level. Thus, for studying meaningfulness of the model, model making and model coefficients evaluation, following statistical hypotheses were considered (Equations (7), (8)).

$$
\begin{gathered}
\left\{\begin{array}{l}
H_{0}: \beta=0 \text { The model is not meaningful } \\
H_{1}: \beta \neq 0 \text { The model is meaningful }
\end{array}\right. \\
\left\{\begin{array}{l}
\text { sig.(p-value })>\alpha=0.05 \rightarrow H_{0} \text { acceptable } \\
\text { sig.(p-value })<\alpha=0.05 \rightarrow H_{1} \text { acceptable }
\end{array}\right.
\end{gathered}
$$

Sig. (p-value) were obtained from Fisher test (Table 4).

Based on Sig (p-value) that was obtained from Fisher test: meaningful

$$
\text { sig }=0.000<\alpha=0.05 \rightarrow R H_{0}
$$




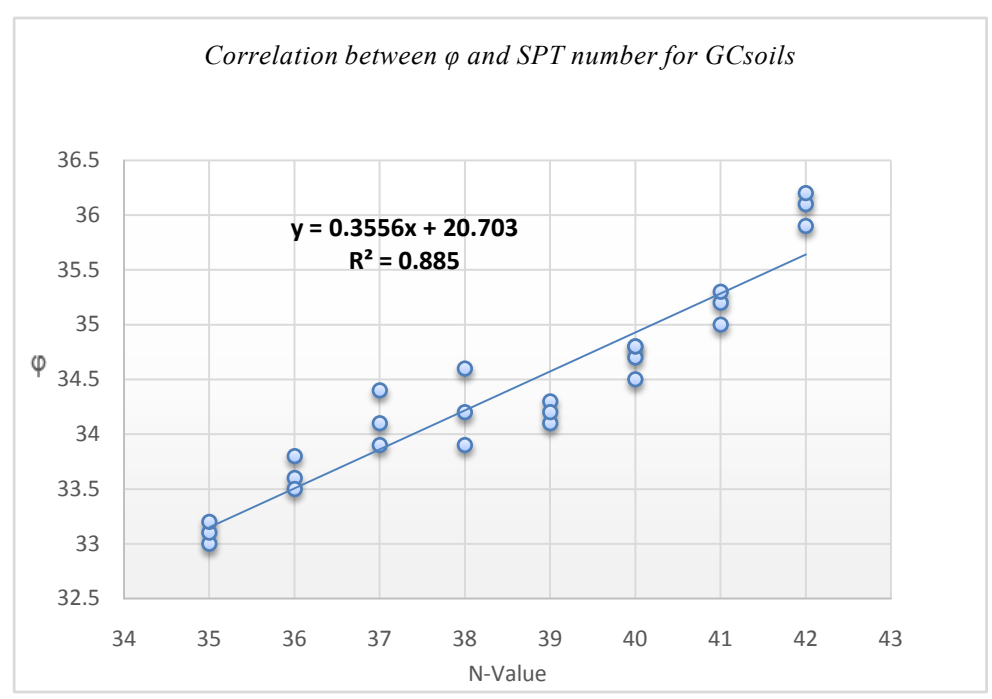

Figure 3. Correlation between $\varphi$ and SPT number for GC soils.

Table 4. ANOVA table for studying the meaningfulness of the model.

\begin{tabular}{|c|c|c|c|c|c|c|}
\hline \multicolumn{7}{|c|}{ ANOVA $^{a}$} \\
\hline \multicolumn{2}{|c|}{ Model 1 for GW Soils } & Sum of Squares & df & Mean Square & $\mathrm{F}$ & Sig. \\
\hline \multirow{3}{*}{1} & Regression & 29.697 & 1 & 29.697 & 316.619 & 0.000 \\
\hline & Residual & 2.157 & 23 & 0.094 & & \\
\hline & Total & 31.854 & 24 & & & \\
\hline \multicolumn{2}{|c|}{ Model 2 for GC Soils } & Sum of Squares & df & Mean Square & $\mathrm{F}$ & Sig. \\
\hline \multirow{3}{*}{2} & Regression & 16.208 & 1 & 16.208 & 177.010 & 0.000 \\
\hline & Residual & 2.106 & 23 & 0.092 & & \\
\hline & Total & 18.314 & 24 & & & \\
\hline
\end{tabular}

${ }^{\mathrm{a}}$ Dependent Variable: PHI; ${ }^{\mathrm{b}}$ Predictors: (Constant), SPT.

According to Table 4, both models are meaningful (Equations (9) and (10)).

Presented equation for GW soils:

$$
\varphi=0.474 S P T+16.188
$$

Presented equation for GC soils:

$$
\varphi=0.3556 S P T+20.703
$$

In Figure 4 and Figure 5, observed friction angle ( $\left.\varphi_{\mathrm{obs}}\right)$ (based on direct shear test) and predicted friction angle $\left(\varphi_{\text {pre }}\right)$ (based on the equations obtained in this research) were compared.

Based on the obtained equations for GC and GW soils, following comparisons with FHWA table values were done:

The results indicate similarity between predicted $\varphi$ values calculated using presented equations in this paper and Peck, Hanson and Thornburn (1974) study; they had also predicted the values of $\varphi$ about 30 to 35 degrees (for this range of SPT numbers). In contrast, the results of this research do not conform to the Meyerhof (1956) study, indicating that he had over-predicted the $\varphi$ values.

\section{Conclusions}

Deriving empirical equations among various geotechnical parameters such as SPT number and internal friction 
angle can be very effective for different purposes such as fast and simple approximate evaluations and reliability rating of laboratory results. In this paper, these correlations were presented for coarse grained and low cohesive soils profiles of Mashhad city. In order to present the mentioned correlations, GC and GW soils with special dry unit weight of 19 to $21 \mathrm{KN} / \mathrm{m}^{3}$ were studied. To avoid weathering effect on results, samples with depths between 4 to $15 \mathrm{~m}$ were used. By narrowing soil type range, depth of sampling and dry unit weight for predicting internal friction angle based on SPT number, two equations were presented. Based on Table 5, FHWA values are similar

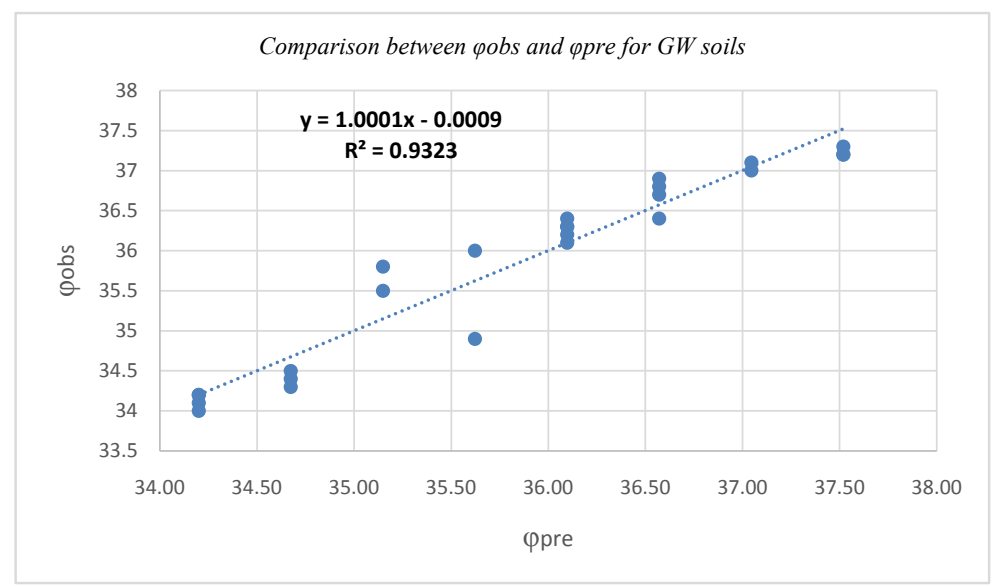

Figure 4. Comparison between $\varphi$ obs and $\varphi$ pre for GW soils.

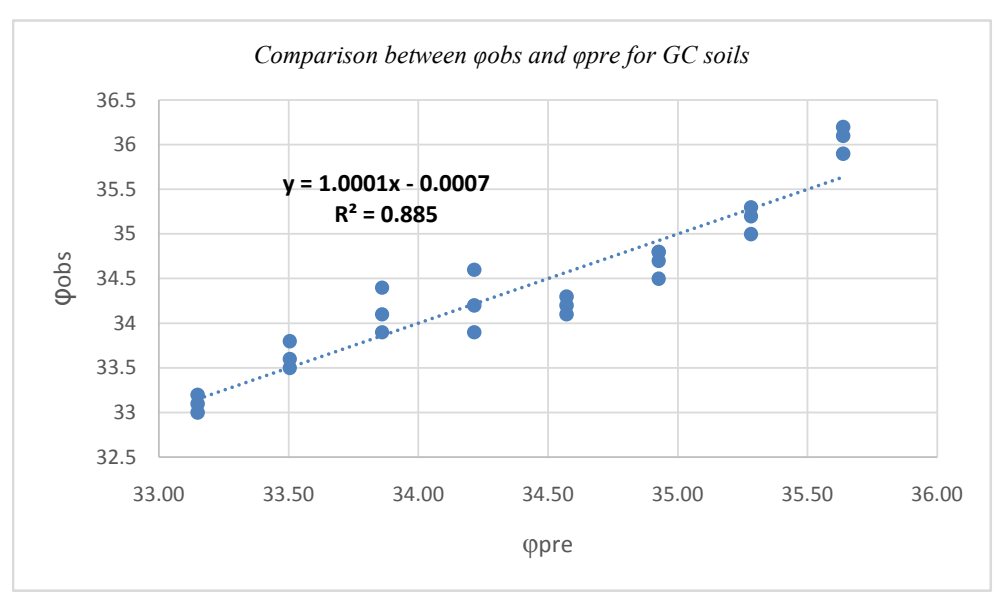

Figure 5. Comparison between $\varphi$ obs and $\varphi$ pre for GC soils.

Table 5. Predicted values of internal friction angle in this research.

\begin{tabular}{|c|c|c|c|c|c|}
\hline & SPT number (in situ test) & $\begin{array}{c}\varphi \\
\text { (by using obtained equation) }\end{array}$ & & $\begin{array}{l}\text { SPT number } \\
\text { (in situ test) }\end{array}$ & $\begin{array}{c}\varphi \\
\text { (by using obtained equation) }\end{array}$ \\
\hline \multirow{8}{*}{ GC soils } & 35 & 33.107 & \multirow{8}{*}{ GW soils } & 38 & 34.1408 \\
\hline & 36 & 33.4558 & & 39 & 34.6259 \\
\hline & 37 & 33.8046 & & 40 & 35.111 \\
\hline & 38 & 34.1534 & & 41 & 35.5961 \\
\hline & 39 & 34.5022 & & 42 & 36.0812 \\
\hline & 40 & 34.851 & & 43 & 36.5663 \\
\hline & 41 & 35.1998 & & 44 & 37.0514 \\
\hline & 42 & 35.5486 & & 45 & 37.5365 \\
\hline
\end{tabular}


to the values of internal friction angle obtained from presented equations in this paper and conform to the results of Peck, Hanson and Thornburn (1974) study. However, the obtained values are mainly lower than the values obtained by Meyerhof (1956).

\section{References}

[1] McGregor, J. and Duncan, J.M. (1998) Performance and Use of the Standard Penetration Test in Geotechnical Engineering Practice. Report of CGPR, Virginia Polytechnic Institute, Blacksburg.

[2] Shioi, Y. and Fukui, J. (1982) Application of N-Value to Design of Foundation in Japan. 2nd ESOPT, Vol. 1, 40-93.

[3] Silva, S.D., Wightman, E.N.R. and Kamruzzaman, M. (2010) Geotechnical Ground Investigation for the Padmamain Bridge. In: Amin, Okui, Bhuiyan, Eds., IABCE-JSCE Joint Conference on Advances in Bridge Engineering-II, Dhaka, Bangladesh, 8-10 August 2010, 427-436.

[4] Hettiarachchi, H. and Brown, T. (2009) Use of SPT Blow Counts to Estimate Shear Strength Properties of Soils: Energy Balance Approach, Journal of Geotechnical and Geoenvironmental Engineering, 135, 25-32. http://dx.doi.org/10.1061/(ASCE)GT.1943-5606.0000016

[5] Jianguo, C. (2012) Correlation Analysis of SPT N Values and Cohesion and Internal Angle of a Clay. Soil Engineering and Foundation, 26, 91-93.

[6] Kaliniski, M. (2011) Soil Mechanics Lab Manual. 2nd Edition, John Wiley \& Sons, Inc., Hoboken.

[7] Mohammad, M. (2013) Reliability of Standard Penetration Test (SPT) in Predicting Properties of Silty Clay with Sand Soil. International Journal of Civil and Structural Engineering, 3, 85-94.

[8] Bowles, J.E. (1988) Foundation Analysis and Design. 4th Edition, McGraw Hill.

[9] Budhu, M. (2011) Soil Mechanics and Foundation. 3rd Edition, John Wiley \& Sons, Inc., Hoboken.

[10] FHWAO-IF-03-17 (2003) Geotechnical Engineering Circular, No.3, Soil Nail Walls.

[11] Aggour, M.S. and Radding, W.R. (2001) Standard Penetration Test (SPT) Correction. Research Report, Civil and Environmental Engineering Department, University of Maryland College Park, Maryland, 20742.

[12] Soil and Structure Consulting Engineers Company (2014) Geotechnical Reports of Vakilabad Region Projects.

[13] Rad, M. (2008) Engineering Statistics and Probabilities. Publications of Hafiz and Tafresh University, Tafresh.

[14] Isotalo, J. (2001) Basics of Statistics. University of Tampere, Tampere. 